\title{
Labyrinthe
}

28 | 2007 (3)

Des Juifs contre l'émancipation

\section{Émancipation, sionisme et Éducation : aux origines du système scolaire israélien}

\section{Aurélia Smotriez}

\section{(2) OpenEdition \\ 1 Journals}

Édition électronique

URL : http://journals.openedition.org/labyrinthe/2903

DOI : $10.4000 /$ labyrinthe.2903

ISSN : 1950-6031

Éditeur

Hermann

\section{Édition imprimée}

Date de publication : 21 décembre 2007

Pagination : 91-103

ISBN : 978-2-9526131-5-6

\section{Référence électronique}

Aurélia Smotriez, «Émancipation, sionisme et Éducation : aux origines du système scolaire israélien », Labyrinthe [En ligne], 28 | 2007 (3), mis en ligne le 01 octobre 2009, consulté le 19 avril 2019. URL : http://journals.openedition.org/labyrinthe/2903; DOI : 10.4000/labyrinthe.2903

Propriété intellectuelle 


\title{
ÉMANCIPATION, SIONISME ET ÉDUCATION: aux origines du système scolaire israélien
}

\author{
Aurélia SMOTRIEZ \\ aurelia.smo@gmail.com
}

Peut-on parler de l'émancipation des Juifs et de son refus en Israël? La question semble a priori ne pas pouvoir s'y poser: l'émancipation, tant dans sa forme politique que culturelle, parait indissociablement liée à la réalité de la vie juive en «diaspora ${ }^{1} »$. Au sens strict, elle désigne un phénomène précis : à partir de la fin du XVIII ${ }^{\mathrm{e}}$ siècle, les Juifs d'Europe occidentale, marginalisés socialement et politiquement dans leurs États de résidence respectifs, deviennent des minorités dont les membres se voient accorder en tant qu'individus l'égalité juridique et politique dont ils étaient jusqu'alors collectivement privés ${ }^{2}$. En Israël, où les Juifs ont collectivement des droits politiques et sociaux du fait de leur souveraineté politique, obtenue en 1948, l'émancipation ne peut garder ce sens proprement politique de processus d'octroi de droits et renvoie dès lors aux conséquences sociales et culturelles qu'il a suscitées. Mais là encore reparaît et s'impose le référent diasporique.

En permettant aux Juifs de «sortir du ghetto», pour reprendre l'expression de l'historien Jacob Katz ${ }^{3}$, et d'investir des domaines sociaux et professionnels qui leur étaient jusqu'alors interdits ${ }^{4}$, l'émancipation politique dont ils ont été les bénéficiaires a confronté les Juifs à l'alternative posée par une «émancipation culturelle» dont ils sont désormais

1. Le terme de «diaspora» doit être replacé dans son contexte historiographique, celui d'un terme générique dont la fonction, selon les lectures religieuse puis sioniste, est de distinguer le centre («Eretz Israel») de la périphérie (le reste du monde, «l'Exil» où vivent les Juifs). Voir infra.

2. La première mesure est prise sous la Révolution française, dans l'édit d'émancipation de 1791 qui affirme l'égalité de tous devant la loi et octroie aux Juifs des droits de citoyens.

3. Voir Jacob Katz, Out of the Ghetto. The Social Background of Jewish Emancipation, Cambridge, Harvard University Press, 1973, traduction française Hors du ghetto. L'émancipation des Juifs en Europe. 1770-1870, Paris, Hachette, 1984.

4. Politique, professions libérales, arts, haute finance... Voir Shlomo Avineri, Histoire de la pensée sioniste, Paris, Jean-Claude Lattès, 1982, p. 13-26. 
les acteurs: s'affranchir des règles fondées sur l'encadrement communautaire religieux, ou continuer à les respecter; accepter l'influence des processus de modernisation et le contact avec la société majoritaire non juive, ou chercher au contraire à s'en isoler pour préserver les structures sociales traditionnelles. Dès les premières mesures d'émancipation, la question de l'éducation, autrement dit de la socialisation des générations suivantes à l'une ou l'autre de ces deux options idéologiques, est l'un des principaux enjeux qui cristallisent cette alternative en un conflit interne aux communautés juives. Les adeptes du mouvement de la haskala, l'équivalent juif d'origine allemande des Lumières ${ }^{1}$, établissent des écoles qui constituent une véritable «révolution ${ }^{2}$ » dans la conception juive traditionnelle de l'éducation, jusque-là dominée par la structure scolaire du 'heder ${ }^{3}$ - école élémentaire pour garçons dédiée principalement à l'apprentissage des textes religieux. L'enseignement des connaissances scientifiques modernes, de la culture humaniste et laïque de la société non juive, et du judaïsme redéfini dans le sens d'une culture plus profane que religieuse ${ }^{4}$ représente aux yeux des maskilim l'outil indispensable de la modernisation sociale, économique et culturelle des Juifs et la clef d'une émancipation réussie. Les Juifs d'Europe de l'Est et de Russie, bien que vivant sous des régimes autoritaires et donc coupés des processus de libéralisation politique, n'en ont pas moins été affectés par des bouleversements - pogromes antisémites, idéaux révolutionnaires socialistes, pénétration des idées de la haskala, mouvement des nationalités des Empires russe et austro-hongrois, etc. qui ont posé pour eux le problème de l'émancipation culturelle dans des termes comparables à ceux de l'alternative entre «modernisme» et «traditionalisme $»^{5}$ connue par leurs pairs occidentaux. Hormis les deux

\footnotetext{
1. Dès le dernier quart du XVIII ${ }^{e}$ siècle, les maskilim, partisans de la haskala, plaident en faveur de la séparation des sphères privée et publique, et proposent au Juif d'«être juif chez lui et homme dans le monde». Voir Élie Barnavi, Une histoire moderne d'Israël, Paris, Flammarion, 1988, p. 14.

2. Voir Rachel Elboïm-Dror, «Les origines du système éducatif isaélien», dans 50 ans d'éducation en Israël, éd. Peled Elad, Jérusalem, ministère de l'Éducation, de la Culture et des Sports, 1999, p. 27 (hébreu).

3. Voir Michael Rosenak, «Jewish fundamentalism in Israel education», dans Fundamentalisms and Society: Reclaiming the Sciences, the Family, and Education, éd. Martin E. Marty et R. Scott Appleby S., Chicago, University of Chicago Press, 1993, p. 374-414.

4. L'hébreu n'y est plus seulement la langue sacrée des Écritures, mais une langue enseignée à des fins de communication (écrite) des savoirs modernes (sciences, histoire, littérature...).

5. Le sociologue Menachem Friedman qualifie de «moderne» cette identité traditionaliste aussi appelée «orthodoxie», dans la mesure où l'effort pour maintenir la tradition religieuse telle qu'elle était
} 


\section{Émancipation, sionisme et éducation}

pôles extrêmes que sont l'abandon total de la religion juive (l' «assimilation») et la défense intransigeante du mode de vie religieux (l' " orthodoxie»), les réponses apportées à cette question en diaspora ont toutes en commun, malgré leur diversité considérable, le désir de maintenir l'identité juive tout en refusant le séparatisme total avec la majorité non juive ${ }^{1}$ - la diversité tenant aux définitions, différentes selon les réponses, du séparatisme social et du contenu culturel de l'identité juive souhaités.

L'émancipation culturelle elle-même semble donc liée à la condition des Juifs comme minorité vivant dans une communauté politique non juive, et paraît, à ce titre, difficile à concevoir en Israël, où les Juifs sont majoritaires. Pour autant, l'absence de la dichotomie minorité juive/majorité non juive en Israël n'y modifie pas les termes de la question centrale qui se pose aux Juifs depuis l'émancipation culturelle du XIX siècle et qui continue aujourd'hui encore à s'y poser, celle de la place et de la fonction de la religion juive dans la réalité sociale moderne. Avec l'apparition du sionisme, à la fin du XIX ${ }^{\mathrm{e}}$ siècle, qui dessine puis matérialise en 1948 la perspective d'un État juif, ce sont les notions de minorité et de majorité ainsi que leurs frontières qui se voient redéfinies. Si l'opposition entre «traditionalistes» et «modernistes» au sein du monde juif précède d'un siècle le sionisme, le nationalisme juif vient néanmoins l'aggraver puis la cristalliser à travers ce qui sera qualifié dans la culture politique sioniste puis israélienne de «clivage» entre «religieux» et «laïcs». C'est autour de ce clivage que se recrée en Israël la frontière entre minorité et majorité.

Notons cependant que cette frontière, ainsi que les significations respectives de ces deux catégories ont évolué elles aussi au cours des deux siècles qui suivent l'émancipation et avec l'histoire de la construction nationale juive. Ceux qui constituent une part largement majoritaire de la population juive mondiale à la fin du $\mathrm{Xx}^{\mathrm{e}}$ siècle - les Juifs « orthodoxes »-deviennent au cours de la première moitié $\mathrm{du} \mathrm{XX}^{\mathrm{e}}$ siècle pro-

vécue et/ou représentée par les Juifs jusqu'au début des processus de sécularisation est une réaction délibérée de différents courants du judaïsme traditionnel qui se rassemblent pour faire front face aux «dangers» de la modernité. Voir La Société ultra-orthodoxe: origines, tendances et évolutions, Jérusalem, Jerusalem Institude for Israel Studies, 1991, p. 6 (hébreu).

1. Il s'agit là du plus petit dénominateur commun entre des mouvements aussi différents que la haskala, la Réforme, la néo-orthodoxie, l'Alliance israélite universelle, le Bund ou le sionisme. Voir le glossaire. 
gressivement minoritaires sur le plan culturel et démographique, en raison même de l'expansion de l'idéologie sioniste qui, au milieu du siècle, est le courant politique le plus actif et structuré dans le monde juif. Au sein du mouvement sioniste, les Juifs dits «laïcs» sont à la fois culturellement hégémoniques et quantitativement majoritaires - d'abord à l'Organisation sioniste mondiale (OSM) en diaspora puis progressivement en Palestine, et en Israël. Dès l'origine, en effet, le nationalisme juif s'énonce en termes de sécularisation, articule une volonté de rupture à l'égard de la religion juive traditionnelle - même s'il est aussi fondamentalement porteur d'une ambiguiité à son égard: celle qui consiste à prendre pour support de la mobilisation nationaliste les sources et les symboles du judaïsme ${ }^{1}$.

Contrairement aux tenants de la haskala allemande, pour qui le judaïsme traditionnel est le signe d'un archaïsme qui ne peut que freiner l'émancipation et l'insertion de l'individu juif dans la société moderne, les sionistes voient dans la religion non plus seulement un obstacle à la modernisation, mais le symbole même de l'existence juive en diaspora dont ils constatent l'impasse ${ }^{2}$. Pour Herzl, la régénération ${ }^{3}$ des Juifs doit passer autant par une émancipation des cadres communautaires religieux que par une émancipation collective «nationale» pensée avant tout en termes politiques et territoriaux - l'objectif étant pour le père du sionisme d'assurer la «normalisation» politique des Juifs en œuvrant à la constitution d'un État souverain comme refuge ${ }^{4}$. En se fondant sur une conception non plus religieuse mais ethnique du judaïsme, mobilisée autour du référent territorial de la «Terre d'Israël»,

1. Loin de se résoudre dans le processus de construction nationale et étatique, cette ambiguïté s'y complexifie. Voir notamment Baruch Kimmerling, «Between the primordial and the civil definitions of the collective identity: Eretz Israel or the State of Israel?», dans Comparative Social Dynamics, éd. Erik Cohen, Moshe Lissak et Uri Almagor, Boulder, Westview Press, 1985, p. 262-283; et «Religion, nationalism and democracy in Israel», dans Constellations, 6, 3, 1999, p. 339-363.

2. En raison notamment de la recrudescence de l'antisémitisme dans les sociétés européennes du dernier quart du XIX ${ }^{e}$ siècle.

3. La question de la régénération est liée à celle de l'émancipation dès les premières mesures politiques prises en Europe. Les «émancipateurs» octroient aux Juifs les droits politiques moins par sympathie pour le judaïsme que par volonté de «corriger les Juifs», selon le mot de l'abbé Grégoire. L'émancipation politique des Juifs, leur inclusion dans la communauté nationale se veut le premier pas vers leur régénération.

4. C'est en cela qu'il se distingue de l'aspiration religieuse du «retour à Sion», même si le sionisme finit par choisir la Palestine comme territoire-cible, en raison de l'attrait sentimental fort que Jérusalem exerce sur les Juifs que le sionisme tente de rallier, et qui lui sont au départ majoritairement hostiles. 


\section{Émancipation, sionisme et éducation}

le sionisme entend écarter le risque de l' «assimilation», de la dilution de l'identité dans une société majoritaire non juive, et établir alors la possibilité d'adopter une nouvelle version de l'identité juive, pour lui donner un contenu culturel différent de celui de la diaspora. Là encore, l'école se fait d'emblée le principal réceptacle et vecteur de l'idéologie sioniste, comme des oppositions qu'elle suscite. Le premier Congrès sioniste mondial, réuni à Bâle en 1897, donne son impulsion décisive au développement des activités sionistes dans le champ éducatif juif européen. Celles-ci prennent appui sur l'institution scolaire fondée par la haskala russe, le «'heder metukan» (le «'heder réformé») ${ }^{1}$, qui, avec l'apparition du sionisme, s'intègre à un réseau scolaire désormais appelé «Tarbut ${ }^{2} »$ («culture» en hébreu). Les écoles de ce réseau constituent autant de foyers d'activités sionistes, et forment les futurs premiers cadres du mouvement.

L'éducation, destinée dans une perspective sioniste à forger le «nouvel homme juif», est considérée comme relevant d'une mission nationale, à laquelle il faut donner une priorité absolue. À cette fin, sont engagés dans le cursus scolaire non seulement la laïcisation de l'hébreu comme langue centrale du dispositif d'enseignement général, mais aussi l'enseignement de la Bible conçu non plus comme texte sacré mais avant tout comme récit de l'histoire du royaume juif antique, présenté comme celle de la «normalité» politique, et légitimant le lien du peuple juif avec la Palestine, appelée Eretz-Israel. Cette relecture dichotomique de l'histoire se fonde sur la doctrine sioniste de la «négation de l'exil» (shlilat ha-galut), qui dévalorise, voire évacue l'histoire des Juifs en diaspora, en opposant un passé lointain et glorieux au passé dit récent de la «dispersion» ou de l' «exil», d'abord marqué par l'absence de souveraineté politique du peuple juif. Pour le sionisme, l'école doit donc se distinguer radicalement du 'heder traditionnel pour devenir l'outil de la transformation d'une société nouvelle à la fois productive et souveraine.

Or, ces perspectives nationalistes constituent pour les Juifs orthodoxes une «révolution» supplémentaire et un danger encore plus into-

\footnotetext{
1. L'intention est claire: il s'agit de garder le lien sémantique ('heder) avec le monde juif traditionnel pour être en mesure de le mobiliser, tout en soulignant clairement l'intention de changement et d'adaptation aux nouvelles formes de scolarité du monde occidental.

2. Voir Shlomo Swirski, Politics and Education in Israel. Comparisons with the United States, New York, Falmer Press, 1999, p. 26.
} 
lérable que celui de la haskala. Le sionisme est en effet considéré comme un «rival hérétique» qui non seulement entend engager un mouvement de retour à la Terre d'Israël sans attendre l'intervention messianique, mais qui en outre s'approprie scandaleusement le patrimoine religieux du judaïsme pour le laïciser ${ }^{1}$. Mais surtout, en niant le judaïsme diasporique, les sionistes dénoncent précisément ce qui est au cœur même de l'existence juive idéale selon la tradition rabbinique, à savoir l'étude talmudique, sacrée pour les autorités traditionnelles, mais rejetée par l'idéologie sioniste qui voit dans cette centralité accordée à l'étude l'une des principales causes de l'absence de travail productif, considérée comme l'affliction majeure de l'existence juive en diaspora ${ }^{2}$. L'opposition des orthodoxes aux processus de modernisation est donc redoublée par l'apparition du mouvement sioniste. C'est avec son développement qu'elle va se structurer durablement et que vont se durcir les termes du conflit avec les «laïcs». Cependant, avec la perspective de la coexistence dans un futur État juif établi par une majorité sioniste, l'enjeu du conflit n'est plus la définition des frontières du groupe ethnique mais celle du contenu culturel de l'identité du groupe.

Pour les orthodoxes, la notion même d'assimilation prend un sens nouveau: elle ne désigne plus tant le risque de la dissolution de l'identité juive dans une majorité non juive, que celui de sa dénaturation au sein d'une société majoritairement composée de Juifs «ethniques», mais porteurs d'une identité inauthentique au regard de sa définition normative. «L'assimilation dans l'État juif» représente un danger accru: celui que les Juifs appartenant encore au monde de la «tradition» n'adhèrent à cette identité majoritaire mais trompeuse, et ne se laissent emporter par la vague de sécularisation apparue avec l'émancipation. Les leaders rabbiniques perçoivent bien la menace que repré-

1. Tout le lexique sioniste emprunte en effet à des notions religieuses qui, du point de vue orthodoxe, sont totalement vidées de leur sens sacré: Eretz-Israel, Aliya («Montée» religieuse vers la Terre Sainte devenue simple émigration des Juifs vers la Palestine), Geulat ha-aretz (la «Rédemption de la Terre», notion à laquelle est donné le sens profane de bonification par le travail agricole), etc.

2. Il en résulte une grande proximité idéologique entre l'impératif sioniste de la «conquête du travail», prôné par A. D. Gordon et celui de la «conquête de l'école» évoqué par Ahad Ha'am, héraut du sionisme culturel. Pour Gordon, la «conquête du travail» ou la «religion du travail» est d'abord une dénonciation du mode de vie improductif des Juifs en diaspora, qu'il qualifie de «peuple parasite», auquel il oppose sa vision d'une «nation juive régénérée par le travail agricole en Palestine». Voir Alain Dieckhoff, L'Invention d'une nation. Israël et la modernité politique, Paris Gallimard, 1993, p. 99 et p. 157. 


\section{Émancipation, sionisme et éducation}

sente l'attrait de cette nouvelle identité laïcisée auprès des masses juives traditionnelles d'Europe de l'Est, «au moment précis où leur situation se dégrade dans ces pays ${ }^{1} \gg$. L'émigration plus tardive de ces Juifs vers la Palestine, dans les années 1920-1930, confirmera à la fois la dimension de refuge et d'alternative que peut représenter le sionisme.

Dès lors s'impose pour les orthodoxes la nécessité d'ériger des frontières internes à la population juive en diaspora même, comme en Palestine $^{2}$. Il s'agit de mettre en place toute une série de stratégies que l'on peut qualifier d' «isolationnistes» ou de «séparatistes $»^{3}$, consistant à dresser des lignes de démarcation symboliques (interdits, distinctions vestimentaires, linguistiques, etc.) entre la communauté traditionaliste et la société juive sioniste désignée comme laïque. L'élève (qu'il soit enfant au 'heder, ou adulte en yeshiva) doit accepter l'autorité des gdolim («grands»), les leaders traditionnels ou charismatiques, qui seuls ont la sagesse et l'autorité nécessaire pour interpréter les textes sacrés, et sont seuls responsables du cursus scolaire ${ }^{4}$. Les murs de l'école sont là encore investis d'un rôle prépondérant dans la mise en place du séparatisme social par les Juifs religieux.

Si la tension entre laïcs et religieux est loin d'être la seule à traverser la collectivité juive en Europe et en Palestine (le corps politique sioniste notamment est lui-même atomisé en de nombreux partis politiques aux idéologies divergentes), elle est certainement l'une des plus âpres. Elle est aussi celle qui, paradoxalement, fait naître le plus de compromis: elle est en effet celle qui sollicite le plus d'efforts pour garantir la mythique «unité du peuple juif» mais surtout la réussite de l'entreprise sioniste. À cet égard, le champ de l'éducation juive est à la fois celui où éclatent ces conflits et celui où s'expérimente le «consociationalisme», pour reprendre le concept du politologue Arend Lijphart

1. Voir Menachem Friedman, op. cit., p. 19.

2. Où vit une communauté juive antérieure à l'apparition du sionisme, appelée le «vieux Yishuv» (par opposition au «nouveau Yishuv» qui désigne la communauté juive sioniste de Palestine avant la création de l'État d'Israël), et dont une partie est constituée d'orthodoxes ashkénazes qui s'opposeront radicalement aux sionistes, et qui seront rejoints par les orthodoxes venus d'Europe de l'Est dans l'entre-deux-guerres.

3. Asher Cohen et Bernard Susser optent pour le terme d'isolationnisme dans leur Israel and the Politics of Jewish Identity. The Secular-Religious Impasse, Baltimore, John Hopkins University Press, 2000, p. 3, tandis que le sociologue israélien de l'orthodoxie religieuse, Menachem Friedman, parle de séparatisme (hitbadlut). Voir Menachem Friedman, op. cit., p. 14.

4. Voir Michael Rosenak, op. cit., p. 382-386. 
abondamment appliqué au Yishuv, mais qui correspond également aux relations entre Juifs laïcs et religieux en Europe dès l'apparition du mouvement sioniste ${ }^{1}$. Le «modèle consociationnel» consiste à tenter de prévenir le conflit politique comme principe des relations entre les différentes communautés (mais non les conflits comme réalité politique), par la mise en œuvre d'un système élaboré de négociations et de concessions que font les différents groupes tout en essayant de maintenir leur intégrité. À travers la défense et le développement de leur système éducatif, l'objectif prioritaire des orthodoxes est donc de recréer un «ghetto» apte à protéger les Juifs des influences modernisatrices mais aussi à combattre les effets de l'émancipation nationaliste.

Ces modalités «défensives» et «offensives» du séparatisme sont toutefois mises en œuvre différemment suivant les courants qui divisent le camp orthodoxe même. De fait, le sionisme n'a pas seulement amplifié l'opposition entre religieux et laïcs, il a aussi accéléré la fragmentation interne à l'orthodoxie ${ }^{2}$, entre un courant en opposition radicale au mouvement sioniste, qualifié d' «ultra-orthodoxe», et certaines autorités rabbiniques, minoritaires, qui acceptent progressivement l'idée du nationalisme juif, et seront qualifiées d' «orthodoxes sionistes », puis de «sionistes-religieuses» dans la culture politique israélienne. Si l'antisionisme reste une valeur centrale dans l'idéologie ultra-orthodoxe, il perd progressivement de sa virulence caractéristique des premiers temps du sionisme et devient de plus en plus difficile à maintenir dans les faits, notamment en raison de l'installation en Palestine dans les années 1930 et 1940 de nombreux orthodoxes fuyant l'antisémitisme européen, et des interactions suscitées avec la société «laïque»du Yishuv - la communauté juive sioniste de Palestine. La création de l'État d'Israël met un terme, sauf pour une minorité radicale d'ultraorthodoxes, à l'utopie du séparatisme absolu avec la société juive «laïque» sioniste.

1. Voir Arend Lijphart, «Consociational Democracy», World Politics, 21, 2, 1969, p. 207-225. Sur l'usage de ce concept, voir entre autres Asher Cohen et Bernard Susser, op . cit; Eliezer Don-Yehiya, «The resolution of religious conflicts in Israel», dans Conflict and Consensus in Jewish Political Life, éd. Stuart A. Cohen et Eliezer Don-Yehiya, Jérusalem, Bar-Ilan University Press, 1986, p. 203-218.

2. Avant l'apparition du sionisme, sous l'effet des processus de modernisation et de sécularisation liés à l'émancipation en Europe, se distinguent déjà au sein de ce front «traditionaliste» deux attitudes : l'une, dite «ultra-orthodoxe», de rejet radical de la modernité; l'autre, appelée «néo-orthodoxe», de méfiance ambivalente à l'égard des savoirs profanes qui s'ajoutent au programme scolaire du 'heder traditionnel mais ne le modifient pas, selon le principe «Torah et culture générale», «Torah im derekh eretz». 


\section{Émancipation, sionisme et éducation}

Mais les frontières entre les Juifs «ultra-orthodoxes» et les «sionistes-religieux » telles qu' on les entend dans l'Israël contemporain, ne correspondent pas à la réalité sociologique du début du $\mathrm{XX}^{\mathrm{e}}$ siècle ${ }^{1}$. La séparation entre ces deux courants est le résultat d'une construction historique, elle-même liée en grande partie aux enjeux de l'éducation, qui couvre la première moitié $\mathrm{du} \mathrm{XX}^{\mathrm{e}}$ siècle. Forts de leur statut majoritaire au sein du monde juif au début du siècle, les orthodoxes aussi bien sionistes qu' antisionistes, adoptent dans un premier temps une stratégie de «séparatisme offensif». Pour ceux qui adhèrent à l'OSM, tel le parti orthodoxe Mizrahi créé en 1902 dans le but de mener cette lutte «de l'intérieur», il s'agit d' «accroître le rôle des Juifs religieux dans le mouvement sioniste, et prévenir les décisions contraires au judaïsme traditionnel $»^{2}$, dans l'espoir, à terme, de concurrencer la définition par les sionistes laïcs des contenus de l'éducation dans le futur État juif et d'y faire triompher une vision du monde religieuse. Le séparatisme scolaire des orthodoxes antisionistes, représentés à partir de 1912 par le parti Agoudat Israel, est lui aussi de type «offensif»: non pas au sens où les ultra-orthodoxes entendent influencer un système scolaire qu'ils ne reconnaissent pas, mais dans la mesure où, se considérant comme les seuls représentants légitimes du peuple juif, ils tiennent leurs institutions scolaires pour les seules sources de diffusion de l'éducation juive authentique.

À mesure que le camp sioniste laïc impose sa domination à partir des années 1920, en diaspora à l'OSM, puis en Palestine même, ce que les Juifs religieux ne revendiquent plus la définition des objectifs pédagogiques de l'Etat juif $^{3}$, mais davantage une autonomie destinée à défendre leurs institutions scolaires contre les influences sionistes laïcistes. Cette évolution vers un «séparatisme défensif» se produit chez les orthodoxes sionistes comme chez les ultra-orthodoxes mais selon

\footnotetext{
1. Ainsi en hébreu, les orthodoxes et ultra-orthodoxes sont confondus pendant au moins les deux premières décennies du siècle, sous le même vocable de «'haredim», qui est d'abord le qualificatif générique des tenants de l'orthodoxie juive, qu'ils adhèrent au sionisme ou le refusent radicalement. C'est seulement à partir des années 1930, et plus encore après la Seconde Guerre mondiale et la création de l'État d'Israël que l'adjectif «'haredi» désignera exclusivement les ultra-orthodoxes opposés au sionisme, ainsi que le rappelle Menachem Friedman, op. cit., p. 9.

2. Voir Charles S. Liebman et Eliezer Don-Yehiya, Religion and Politics in Israel, Bloomington, Indiana University Press, 1984, p. 63.

3. Même si cet objectif ne disparaît jamais tout à fait des discours religieux, et réapparait sous des formes plus ou moins explicites selon les périodes.
} 
deux modalités distinctes, et à trente années d'intervalle. Dans les deux cas, elle concerne les écoles religieuses établies en Palestine. Les premiers à accepter le principe de l'autonomie et à renoncer au pouvoir de définition des buts éducatifs collectifs, sont les orthodoxes sionistes. L'évolution historique du rapport de forces en faveur du camp laïc les conduit même à revendiquer assez tôt l'autonomie pour leurs établissements de Palestine. C'est en 1920 que le parti Mizrahi obtient la reconnaissance formelle par l'OSM au Congrès sioniste de Londres, de son réseau scolaire et de son autonomie pédagogique ${ }^{1}$, au terme de négociations complexes avec des dirigeants sionistes laïcs pour la plupart peu enclins à renoncer au monopole sur l'interventionnisme dans le champ éducatif que leur octroie leur suprématie politique. Mais c'est dès 1902 que la perspective d'un compromis sur les buts de l'éducation sioniste est débattue, lors de la Conférence des sionistes russes à Minsk au cours de laquelle se manifestent de vifs antagonismes entre orthodoxes et laïcs sur la question scolaire. Dans la crainte de la sécession des membres religieux du mouvement sioniste ${ }^{2}$, l'un des principaux théoriciens du sionisme, Ahad Ha'am, propose aux uns et aux autres d'adopter le principe de la «séparation dans l'unité ${ }^{3}$ ». Il suggère que la «renaissance nationale» soit définie comme l'objectif commun des membres laïcs et religieux du sionisme, mais que la méthode pour y parvenir soit laissée au libre choix de chaque camp. Si elle est rejetée dans un premier temps par les deux camps, cette proposition a néanmoins une importance capitale, car c'est la première fois qu'est formulée la valeur politique accordée au consensus, tenu pour garant de l'unité communautaire, et pour laquelle les dirigeants sionistes de diaspora, puis ceux du Yishuv, et enfin d'Israël, sont prêts à payer le prix de la segmentation interne en renonçant à la scolarisation commune des Juifs. De son côté, le parti orthodoxe Mizrahi, divisé sur cette question,

1. Voir Rachel Elboïm-Dror, «Les origines du système éducatif israélien»..., op. cit., p. 23

2. Les débats sur la nature de l'éducation divisent tous les premiers Congrès du mouvement. Herzl tente d'éviter que ces questions ne soient mises à l'ordre du jour, mais la victoire, au cinquième Congrès sioniste de 1901, de la résolution sur «l'éducation nationale» présentée par la «Fraction démocratique», parti représentant le camp laïc militant à l'OSM, suscite la création en 1902 du parti politique Mizrahi.

3. «Yikhud bi'tzad hafrada» en hébreu. Voir Joseph Schwartzwald, L'Éducation publique-religieuse en Israël, Ramat-Gan, Presses de l'université de Bar-Ilan, 1990, p. 16, (hébreu), et Shmuel Shamai, " "Cultural shift": the case of Jewish religious education in Israel», in British Journal of Sociology of Education, vol. 21, n 3 , septembre 2000. 


\section{Émancipation, sionisme et éducation}

la tranchera une décennie plus tard, lorsque ses membres, qui refusent le plus radicalement toute intervention de l'OSM dans les activités culturelles même au prix d'une autonomie reconnue, font sécession pour créer en 1912 un nouveau parti religieux, le parti ultra-orthodoxe Agoudat Israel. C'est en acceptant la «séparation dans l'unité» que le Mizrahi d'après 1912 devient pleinement un parti orthodoxe sioniste, le futur Parti national religieux.

En revanche, le séparatisme des ultra-orthodoxes du parti antisioniste Agouda est à son apogée dans les années 1920. Il adopte une modalité d'autant plus offensive que la souveraineté politique sur la Palestine est alors celle du Mandat britannique, qui perpétue le système du millet ottoman et reconnaît les communautés sur une base religieuse. Cette reconnaissance, ainsi que l'aspect volontaire de l'adhésion des différents groupes aux institutions politiques et sociales du Yishuv, facilite donc l'autonomisation de la communauté orthodoxe antisioniste de Palestine ${ }^{1}$. Cependant, à partir du milieu des années 1930, la coopération avec les institutions politiques sionistes se développe; c'est notamment sous l'effet de l'émigration vers la Palestine de nombreux Juifs qui, en raison de la montée des périls, quittent les communautés traditionalistes d'Europe de l'Est. L'idée de l'établissement d'un État juif, au moins comme refuge, est acceptée de fait par un nombre croissant d'ultraorthodoxes. Malgré ces évolutions, ce n'est qu'au lendemain de la Seconde Guerre mondiale et à la veille de la création de l'État d'Israël, que les responsables laïcs et ultra-orthodoxes font des concessions mutuelles. Agoudat Israel est en effet considérablement affaibli après la Shoah, sur un plan démographique, financier, spirituel, etc.: la reconnaissance de l'ampleur du désastre rend le coût du maintien d'un séparatisme offensif exorbitant. Les dirigeants sionistes laïcs quant à eux ont besoin du soutien le plus large possible à la veille du débat aux Nations unies devant décider de l'établissement d'un État juif en Palestine et cherchent à éviter que le parti antisioniste Agouda ne plaide en défaveur de cet objectif. Dans la lettre dite du «statu quo $^{2} »$, document de 1947 appelé à avoir une importance capitale dans l'histoire politique israélienne et dans le mode de gestion consociationnel des relations

1. Voir Joël Migdal, Through the Lens of Israel. Exploration in State and Society, Albany, State University of New York Press, 2001.

2. Voir Ilan Greilsammer, Israël. Les hommes en noir, Paris, Presses de la FNSP, 1991, p. 47 et $s q$. 
entre laïcs et religieux, sont formulées un certain nombre de garanties accordées aux Juifs ultra-orthodoxes par la direction sioniste laïque, sur le respect du shabbat et de la kashrut dans les institutions publiques, mais aussi sur l'autonomie éducative des écoles ultra-orthodoxes dans le nouvel État. Avec le vote de la loi d'orientation générale sur l'Éducation nationale en 1953, qui définit le cadre juridique du système scolaire israélien, cette autonomie des écoles affiliées au parti Agouda est non seulement garantie mais obtient une reconnaissance juridique, trente ans après celle accordée aux sionistes-religieux au Congrès de Londres.

Le système scolaire du nouvel État institutionnalise ainsi sa propre segmentation en fonction du clivage entre laïcs et religieux ${ }^{1}$, mais aussi entre religieux mêmes. Trois types de scolarisation sont reconnus officiellement, définis par le rapport que les différents établissements entretiennent - explicitement - avec la religion, et - implicitement avec l'idéologie sioniste. Les écoles sont ainsi sionistes-laïques, sionistes-religieuses, ou religieuses non-sionistes, dites ultra-orthodoxes. Si les deux premiers types de scolarisation octroient aux écoles un statut d'éducation publique, le troisième relève d'un statut juridique particulier: ni privé, ni public, il jouit cependant d'une autonomie pédagogique et administrative à l'égard du ministère de l'Éducation limitée en droit mais quasi complète dans les faits, tout en bénéficiant du soutien financier presque total de l'État. Quant à l'éducation sioniste-religieuse, si son caractère public lui permet de bénéficier des financements de l'État exactement au même titre que les écoles laïques, il ne lui en accorde pas moins également une large indépendance dans la direction pédagogique et administrative de ses établissements. À l'heure où triomphe le camp laïc sioniste, où la création de l'État d'Israël le dote formellement de son hégémonie culturelle et politique, les Juifs orthodoxes devenus minoritaires tout au long de la première moitié du $\mathrm{XX}^{\mathrm{e}}$ siècle, voient leur stratégie séparatiste - devenue défensive - légitimée et institutionnalisée par la majorité elle-même. Ce sont ces fondations juridiques qui leur permettront, après 1967 et principalement dans les années 1980 et 1990, d'adopter une nouvelle attitude offensive: sionistes-religieux et ultra-orthodoxes revendiquent ces dernières années le renforcement du rôle et de la place de la tradition religieuse juive

1. Qui vient après la segmentation première des systèmes scolaires ségrégés juif et arabe. 


\section{Émancipation, sionisme et éducation}

dans l'ensemble du système scolaire israélien, y compris au sein de l'éducation publique-laïque; une partie des ultra-orthodoxes obtient la reconnaissance en 1990 d'un nouveau système scolaire religieux prosélyte, affilié au parti ultra-orthodoxe séfarade Shas.

Si le «clivage laïcs-religieux» est un de ceux où ce principe «consociationnel» agit de manière récurrente, c'est en raison du lien intime, de la tension inhérente au nationalisme juif entre religion et politique, qui favorisent cette culture du consensus et du compromis. En effet, au-delà des avantages matériels que peuvent retirer les religieux ou les laïcs de la pratique consensuelle (ressources pour les uns, stabilité politique pour les autres), cette indétermination garantit aux laïcs comme aux religieux (sionistes et non-sionistes), la légitimité de leur appartenance à la collectivité, sur une base nationale (sioniste) ou religieuse (juive). 